\title{
La evaluación de la formación permanente. Un estudio realizado sobre acciones de capacitación desarrolladas por el Ministerio de Educación de Córdoba, Argentina
}

\author{
Horacio Ferreyra \\ Doctor en Educación \\ Universidad Católica de Córdoba, Argentina \\ Docente e investigador de la \\ Universidad Católica de Córdoba Unidad Asociada CONICET \\ Universidad Nacional de Villa María \\ ITEC-Guadalajara, México. Universidad Santo Tomás, Colombia \\ Subsecretario de Estado de Promoción de Igualdad y Calidad \\ Educativa del Ministerio de la Provincia de Córdoba \\ hferreyra@coopmorteros.com.ar / www.horacioaferreyra.com.ar
}

\author{
Fabiana Castagno \\ Lic. en Ciencias de la Educación \\ Universidad Nacional de Córdoba, Argentina \\ Docente e investigadora de la Escuela de Ciencias de la Información, \\ Universidad Nacional de Córdoba y Universidad Católica de Córdoba \\ Unidad Asociada CONICET \\ Miembro del Equipo Técnico de la Subsecretaría de Estado de \\ Promoción de lgualdad y Calidad Educativa del Ministerio de la \\ Provincia de Córdoba \\ Integrante del equipo de Investigación de Educación Secundaria \\ Unidad Asociada CONICET- \\ Universidad Católica de Córdoba-Facultad de Educación \\ fabianacastagno@hotmail.com
}

Marta Ruiz

Analista en Sistemas de Computación

Miembro del Equipo Técnico de la Subsecretaría de Estado de

Promoción de Igualdad y Calidad Educativa del Ministerio de la

Provincia de Córdoba

mruiz95@gmail.com
Alejandra Salgueiro

Magíster en Educación. Licenciatura en Ciencias de la Educación Universidad Nacional de Buenos Aires

Docente e investigadora de profesora de Taller de Práctica Docente Integrante del Equipo de Formación de Directores de Institutos Superiores INFD - Ministerio de Educación. Miembro de Equipo de la Subsecretaría de Estado Promoción de Igualdad y Calidad Educativa del Ministerio de la Provincia de Córdoba asalguei@live.com.ar

Artículo de reflexión recibido el 27 de marzo de 2015; aprobado el 18 de mayo de 2015.

\section{Cómo citar este artículo:}

Ferreyra, H., Castagno F. y Ruíz, M. (2015). La evaluación de la formación permanente. Un estudio realizado sobre acciones de capacitación desarrolladas por el Ministerio de Educación de Córdoba-Argentina. Espiral, Revista de Docencia e Investigación, 5(1), 61-76.

\section{Resumen}

Este artículo de reflexión presenta un conjunto de nociones y análisis acerca del valor de la evaluación en la construcción de saberes sobre las políticas de formación y desarrollo profesional docente. Para ello, se describe un estudio evaluativo de las acciones de formación docente continua llevado a cabo por un equipo de trabajo de la Subsecretaría de Educación de la Calidad e Igualdad Educativa del Ministerio de Educación, de la provincia de Córdoba, Argentina. Tiene como objetivo relevar información sobre las valoraciones y percepciones que los destinatarios - participantes de las acciones formativasmuestran sobre las ofertas de desarrollo profesional brindadas por este Ministerio durante el período 2010-2012.

La evaluación es concebida en el estudio no solo como herramienta de control sino fundamentalmente en su dimensión epistémica y ética. La estrategia metodológica previó la combinación de abordajes cualitativos y cuantitativos. Se utilizaron como instrumentos relatos escritos, cuestionarios auto administrados y entrevistas grupales. Los resultados obtenidos permiten identificar un conjunto de rasgos destacados como significativos por los participantes en sus experiencias formativas, en aquellas acciones de desarrollo profesional que previeron/contemplaron: intercambio y diálogo con pares, referencia explícita a la propia práctica, anclaje de la propuesta en la escala institucional del trabajo docente/ colaborativo, sinergia con las necesidades y problemáticas de la institución, nivel y región en el cual desempeña su labor.

Palabras clave: Educación, evaluación, formación continua, valoración y percepciones. 


\section{Abstract}

This reflection paper presents a set of notions and analysis on the value of assessment in the construction of knowledge about training policies and teacher professional development. To do this, an evaluation study of the actions of continuous teacher training conducted by a team of the Sub secretary of Education of Quality and Educational Equality of the Ministry of Education of the province of Córdoba-Argentina is described. It aims to note the information about the assessments and perceptions that the participants of the training activities show about professional development offerings provided by the Ministry during the period 2010-2012.

The assessment is conceived in the studies not only as a control tool but mainly in its epistemic and ethical dimension. The methodological strategy foresaw the combination of qualitative and quantitative approaches. Written stories, self-administered questionnaires and group interviews were used as instruments. The results allow to identify a set of features highlighted by participants as significant in their formative experiences in those actions of professional development that were contemplated: exchanges and dialogue with peers, explicit reference to the practice itself, anchoring the proposal on the institutional scale professor/collaborative work, synergy with the needs and problems of the institution, level and / or region in which the professor plays his role.

Keywords: Education, evaluation, training, assessment and perceptions.

\section{Introducción}

En el marco de las políticas de formación continua orientadas al fortalecimiento de los conocimientos y prácticas de los equipos directivos y de los docentes del sistema educativo, el Ministerio de Educación de la Provincia de Córdoba viene desarrollando diversas acciones de desarrollo profesional.

En línea con la necesidad de contar con insumos que posibiliten evaluar el desarrollo junto a posibles impactos de dichas acciones y en consonancia con el interés de construir conocimiento sobre la formación continua, la Subsecretaría de Promoción de Igualdad y Calidad Educativa impulsó dos estudios destinados a evaluar acciones de formación continua desde la perspectiva de los destinatarios en dos períodos diferentes. Uno, abordó el bienio 2008-
2009 y otro el lapso 2010-2012. En este artículo se hará referencia a resultados ${ }^{1}$ del segundo período.

El objetivo central de este estudio fue evaluar las acciones de formación docente continua. Hizo foco en las percepciones y valoraciones de los destinatarios (se consideran destinatarios a los participantes de las acciones formativas) construidas como consecuencia de ser partícipes de la oferta ejecutada en el tramo mencionado. De esta manera, se buscó indagar qué pensaban y qué decían acerca de la experiencia generada en ese marco formativo.

La elección de esta perspectiva se apoya en el reconocimiento del valor de la palabra de los actores involucrados que aportan una mirada particular de alto valor, ya que posibilita una aproximación diferente al objeto de estudio. Sus apreciaciones no solo contribuyen a ofrecer datos sobre lo acontecido en las experiencias de capacitación, también son un insumo fundamental para la problematización de las políticas de formación y analizadores privilegiados de las prácticas educativas en general. Este enfoque se vincula con la concepción de evaluación que sustenta el equipo que ha coordinado el estudio, cuyos rasgos centrales se comparten en el próximo apartado.

En ese sentido, la experiencia misma se sostuvo en una práctica evaluativa que pretendió apartarse de aquellas ideas que la conciben como un monstruo en los términos planteados por Mokate (2000a) quien señala: "Al explorar las razones por las cuales la evaluación ha asumido esta imagen de monstruo, se encuentra que la administración tradicional de lo social se ha caracterizado por un enfoque dirigido a objetivos programáticos u operativos" (p. 4).

1 Para mayores precisiones los documentos "La evaluación de acciones de formación docente continua desde la perspectiva de los destinatarios. Período 2008-2009" y "La evaluación de acciones de formación docente continua desde la perspectiva de los destinatarios. Período 2010-2012" se encuentran disponibles en www.igualdadycalidadcba.gov.ar 


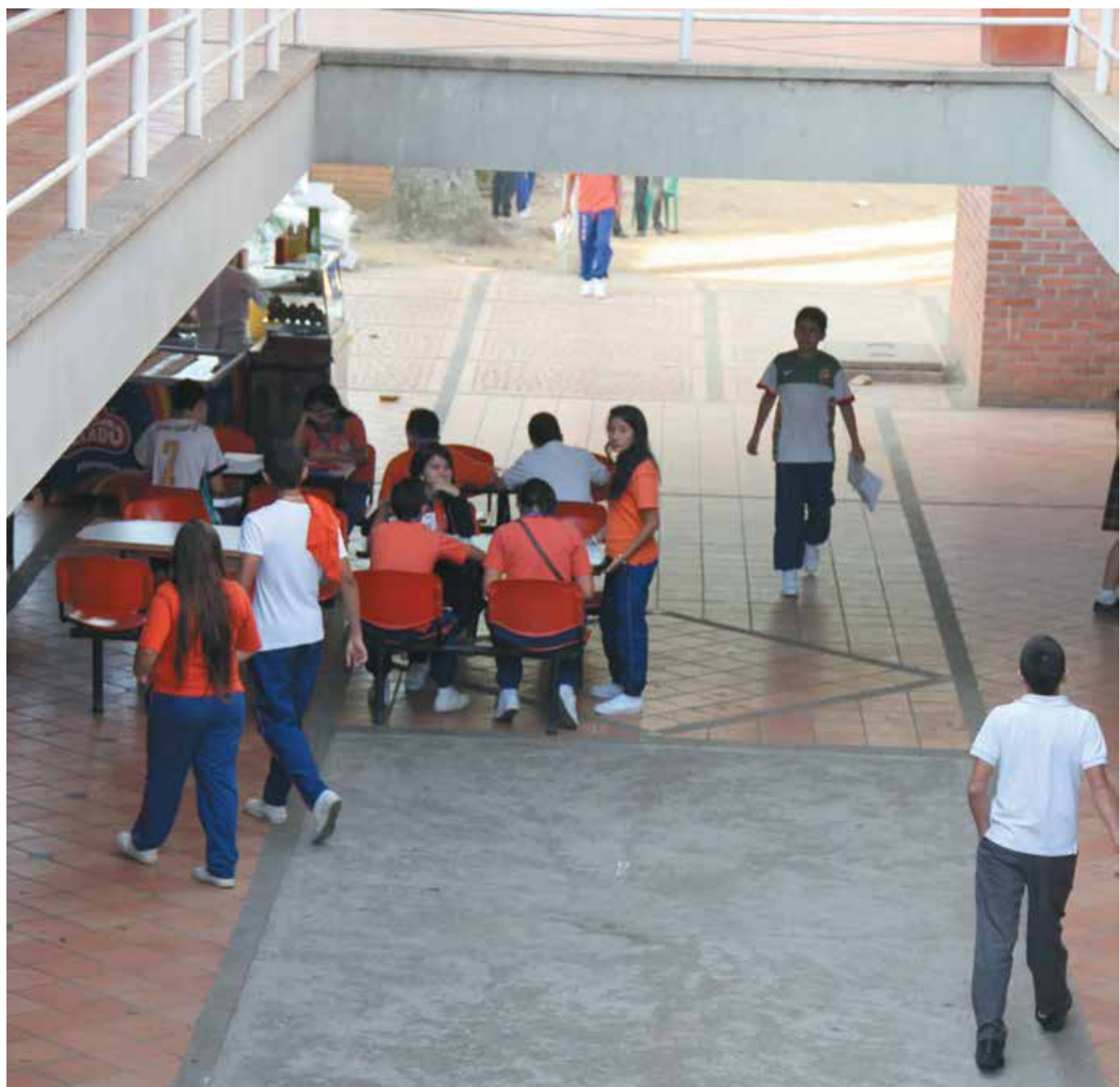




\section{Aspectos teórico-conceptuales}

La evaluación en tanto proyecto, acción, práctica es un acto ético-político, no reducido a la dimensión meramente instrumental y operativa. Significa que todo proceso evaluativo tiene una intencionalidad -explícita o no- que lo orienta en una dirección u otra. Esto implica reconocer que la evaluación, no es una acción neutra, ingenua. Tampoco supone concebirla como algo extraño o ajeno a las políticas públicas, como algo que se acopla a estas. Muy por el contrario, fue concebida como parte constitutiva de sus procesos de diseño y ejecución, para evitar relacionarla solamente a una tarea de rendición de cuentas externa a esta. Mokate (2000b) lo señala como un problema:

(...) a pesar de evaluar y de ser evaluados diariamente, en la esfera de nuestras gestiones públicas, la evaluación es frecuentemente percibida como una intervención no bienvenida. La evaluación formal y rigurosa es frecuentemente interpretada como un ejercicio externo a los procesos que busca afectar y mejorar. A veces se percibe como una auditoría o control impuesto desde afuera (p. 1).

La elección del objeto de evaluación, su recorte y los instrumentos que se diseñan para relevar la información dan cuenta de un posicionamiento teórico, ideológico y ético. La cualidad de ético y político se observa también en el vínculo que establece el investigador con el objeto por investigar, con los sujetos que participan en el tratamiento de los datos (qué se mira, qué se analiza), en su comunicación, en las decisiones que pueden ser tomadas a partir de los resultados obtenidos (Nirenberg, 2008). La presencia y reconocimiento de esta cualidad impugna la pretensión de objetividad de cualquier acción investigativa y evaluativa. No se trata de defender la relatividad absoluta si no de aceptar que las lecturas que puedan hacerse de una realidad particular se realizan desde una determinada perspectiva y marco conceptual que no son los únicos posibles, son unos entre muchos y que su elección implica posicionarse teórica e ideológicamente.
La dimensión ética y política de la evaluación, a su vez, advierte sobre la necesidad de pensar acerca de su sentido, por qué y para qué se evalúa, cuestiones que no siempre se explicitan con claridad en sus objetivos. En este sentido, coincidimos con Fernández Ballesteros (1996) en la necesidad de parte de los equipos que asumen esta tarea de precisar y clarificar el para qué en el plano implícito y explícito a la vez que en vínculo con sus funciones asignadas. Desde nuestra posición, la evaluación es una instancia que provee información que debería interpelar políticas, procesos, prácticas, a fin de problematizarlas $y$, desde esta reflexión, acercar un conocimiento que permita generar y producir transformaciones (Pichardo Muniz, 1993, Pinkasz, et al., 2008).

La evaluación arroja datos que tal vez son del orden de lo conocido, la operación que puede darse es la confirmación de hipótesis y supuestos ya pensados y enunciados. Este es uno de los riesgos cuando se evalúa y también se investiga, mirar con los mismos ojos para verificar y conformar nuestras afirmaciones iniciales. Tomamos la idea de Sandra Nicastro de "revisitar", volver a mirar lo ya mirado, asumiendo que se pude explorar y descubrir aspectos que no fueron visibles hasta el momento sin pretender comenzar de nuevo cada vez. Esta posición altera "la comodidad propia de quien se sostiene en lo ya sabido y en la cualidad inconmovible de ese saber" (Nicastro, 2011, p. 25).

La autora advierte sobre el lugar del tiempo en el acto de revisitar, porque cuando se evalúa se entra en la tensión entre el pasado (hechos y fenómenos que ya han ocurrido), el presente del acto de evaluar y el futuro que se presenta como promesa y un porvenir que puede ser alterado, transformado a partir de un proceso evaluativo de las interpretaciones y análisis que lo habilitan. La interpretación de los datos es considerada como acción de desciframiento y traducción: 
(...) la revelación de elementos y relaciones latentes; que no sigue un procedimiento de traducción término a término, como el desciframiento de un jeroglífico, sino que implica un proceso de construcción y reconstrucción de una situación, justamente para hacer situación de un contexto, y desde allí explicarlo (Nicastro, 2011, p. 41).

En nuestro estudio, al tratarse de datos que se componen de las valoraciones y percepciones de los sujetos, esta perspectiva resulta sumamente potente porque busca descifrar los significados que, a través de sus relatos, los actores participantes del trabajo tienen sobre la educación y, en particular, sobre los procesos de formación continua. En este sentido, el

(...) proceso de acercamiento a lo acontecido a través de los relatos, no tiene fin, siempre puede perfeccionarse y profundizarse. La evaluación permite construir un nuevo relato, una explicación posible o nueva interpretación sobre lo ocurrido, para así recomendar senderos distintos para la acción futura (Nirenberg, 2008, p. 23).

Desde este enfoque, la evaluación se presenta entonces como una oportunidad para pensar, reflexionar, comprender. Nirenberg (2008) sostiene que: "la evaluación sirve también para incrementar el conocimiento a partir de la práctica, ya que evaluar implica transformar la información recolectada en nuevo conocimiento" (p. 20). En consonancia con lo expuesto, en la tarea realizada fue estructurante la significación otorgada a la evaluación como una práctica con potencialidad epistémica. De esta manera, se buscó superar la idea de que solo se evalúa para verificar, contrastar, catalogar, sino como una actitud ante la vida $y$, fundamentalmente, como una herramienta para construir futuro (Pichardo Muñiz, 1993).

La historia sobre las prácticas de evaluación y sus formas muestra tendencias diversas que han hecho foco sobre todo en dos aspectos: los resultados y los procesos, en uno u otro. En diferentes momentos uno prevaleció sobre el otro, sesgándose el análisis y descuidando la complejidad que todo hecho social y educativo supone. Probablemente, y a modo de hipótesis, se podría pensar que el énfasis puesto solo en uno u otro aspecto haya afectado la comprensión, problematización de las situaciones educativas tanto como las posibilidades de intervención y mejora.

Sin embargo, ya hace unos años atrás se viene considerando necesario revisar esa suerte de dicotomía. Las actuales perspectivas en torno a la evaluación proponen enlazar procesos y resultados como forma de superar la reducción en la construcción de los datos implicados en todo proceso evaluativo. Desde esta visión más integral es posible recuperar y visibilizar también la dimensión política comprometida en estas prácticas. El mismo término política convoca a enlazar, relacionar y articular los diferentes componentes/elementos que intervienen en cualquier situación social, dando lugar a una comprensión y explicación que posiblemente genere un nuevo conocimiento y acción. Por lo dicho, en el marco de nuestro estudio ambos datos fueron relevados y puestos en diálogo.

En función de lo expuesto, la experiencia de evaluación objeto de esta presentación pretendió constituirse en sí misma en un aprendizaje colectivo y social que involucró diferentes actores y voces, perspectivas y significados. Se trató de un proceso en el cual la puesta en circulación de los resultados se torna un punto crucial al poner en foco nuevamente sus facetas ética y epistémica. En otras palabras:

la socialización de los hallazgos evaluativos no solo tiene un sentido pedagógico y de construcción de nuevo conocimiento, sino también un sentido ético, en la medida que implica compartir con los actores involucrados en el proceso evaluativo (...) los hallazgos, juicios, intervenciones y recomendaciones para que vuelvan a ser objeto de reflexión, discusión y ajuste (Nirenberg, 2010, p. 6). 


\section{Metodología}

Los actores que han formado parte de este estudio son los destinatarios de las acciones de formación permanente desarrolladas por el Ministerio de Educación, durante 20102012. Participaron del estudio un total de 250 docentes del sistema educativo provincial. Se trata de supervisores, equipos directivos y docentes que desarrollan sus actividades en los diferentes niveles y modalidades educativos.

La estrategia metodológica previó la combinación de abordajes cualitativos y cuantitativos. Se trabajó con relatos escritos, cuestionarios autoadministrados y entrevistas grupales. Con estos instrumentos se realizó el trabajo de campo a efectos de generar datos acerca de las experiencias formativas recibidas por los participantes en función del tiempo transcurrido. Se buscó reconocer demandas de formación para el próximo trienio, como también poder conocer aspectos vinculados a los entornos virtuales en sus procesos de formación continua.

El estudio buscó incorporar un tratamiento comparativo que permitiera identificar cambios y continuidades en las percepciones y valoraciones de los destinatarios con respecto a las acciones de formación y desarrollo profesional ejecutadas en el período 2008-2009. En consecuencia, el trabajo de campo se estructuró en dos etapas con perfiles de parti- cipantes diferenciados. La primera etapa estuvo centrada en un abordaje con destinatarios que debían reunir los siguientes requisitos:

a. participar del estudio desarrollado para el período 2008-2009.

b. desarrollar actividad en el sistema educativo provincial al momento de realizar el trabajo de campo.

c. haber participado de al menos dos instancias de formación en el período 20102012.

d. desempeñar algunos de los siguientes roles en el sistema educativo provincial: supervisor, director o docente.

e. desarrollar sus funciones en diferentes regiones o zonas de la jurisdicción, así como los distintos niveles y modalidades que debían estar representadas en el estudio.

La segunda etapa tuvo por objetivo trabajar en terreno con destinatarios que debían reunir los requisitos c., d. y e. explicitados para la primera etapa.

Las tablas que se presentan a continuación detallan el número de participantes de acuerdo con el cargo o función, nivel o modalidad educativa y lugar de desempeño, para cada una de las etapas en que se organizó el trabajo de campo.

Tabla 1. Participantes entrevistados

\begin{tabular}{|l|c|c|c|}
\hline Nivel/modalidad/disciplina & Primera etapa & Segunda etapa & Total \\
\hline Inicial & 10 & 36 & 46 \\
\hline Primario & 26 & 36 & 62 \\
\hline Secundario & 8 & 38 & 46 \\
\hline Ed. Técnico profesional & 8 & 36 & 44 \\
\hline Superior & --- & 14 & 14 \\
\hline Ed. Especial & 6 & 12 & 18 \\
\hline Ed. Jóvenes y adultos-primario & --- & 8 & 8 \\
\hline Ed. Jóvenes y adultos-secundario & --- & 4 & 4 \\
\hline Ed. Física & 8 & 4 & 12 \\
\hline Total & 66 & 184 & 250 \\
\hline
\end{tabular}




\begin{tabular}{|l|c|c|c|}
\hline Posición & Primera etapa & Segunda etapa & Total \\
\hline Supervisores & 15 & 20 & 35 \\
\hline Directivos & 27 & 62 & 89 \\
\hline Docentes & 28 & 98 & 126 \\
\hline Total & 70 & 180 & 250 \\
\hline
\end{tabular}

Fuente: La evaluación de acciones de formación docente continua desde la perspectiva de los destinatarios. Período $2010-2012$ (p. 6).

Tabla 2. Lugar de desempeño por región-departamento

\begin{tabular}{|l|c|c|c|}
\cline { 2 - 4 } \multicolumn{2}{c|}{} & \multicolumn{2}{c|}{ Participantes entrevistados } \\
\cline { 2 - 4 } \multicolumn{1}{c|}{} & Primera etapa & Segunda etapa & Total \\
\hline Capital & 25 & 37 & 62 \\
\hline Río Primero - Río Segundo - San Justo & 2 & 26 & 28 \\
\hline Tercero Arriba - Gral. San Martín - Unión - Marcos Juárez & 12 & 26 & 38 \\
\hline Río Cuarto - Juárez Celman - Pte. R. Sáenz Peña - Gral. Roca & 6 & 20 & 30 \\
\hline Punilla - Colón - Santa María - Calamuchita & 6 & 24 & 20 \\
\hline Ischilín -Totoral - Sobremonte - Río Seco - Tulumba & 4 & 16 & 30 \\
\hline Cruz del Eje - Minas - Pocho - San Alberto - San Javier & 10 & 20 & 16 \\
\hline Distintos departamentos del interior & 5 & 11 & 250 \\
\hline Total & 70 & 180 & 30 \\
\hline
\end{tabular}

Fuente: La evaluación de acciones de formación docente continua desde la perspectiva de los destinatarios. Período 2010-2012 (p. 6).

Las valoraciones y percepciones construidas por los destinatarios en torno a las acciones formativas fueron indagadas sobre la base de dos ejes temáticos:

El primero estuvo asociado con el análisis de cómo fueron las experiencias formativas en el marco de acciones ofrecidas por el Ministerio de Educación de la Provincia. Para ello se delimitaron tres momentos en torno a los cuales describir esas experiencias: el de acceso, de inicio a y de formación en servicio; el de desarrollo de las acciones formativas, y el de apropiación - socialización de la experiencia.

El segundo eje temático estuvo organizado en torno a demandas y otras cuestiones de la formación continua - permanente. Para el abordaje de este tópico se establecieron como dimensiones de análisis: demandas de formación en servicio en el corto plazo; incorporación de nuevas tecnologías de la información y la comunicación en sus procesos de formación profesional; acceso - consulta de documentos y materiales de lectura producidos o sistematizados por el Ministerio y, finalmente, formación en servicio brindada por otros oferentes.

Los ejes temáticos y dimensiones expuestos dieron lugar a la definición y especificación de categorías de análisis que se detallan en la siguiente tabla: 


\section{Tabla 3. Dimensiones y categorías}

\begin{tabular}{|c|c|c|}
\hline & Dimensiones & Categorías \\
\hline \multirow{9}{*}{ 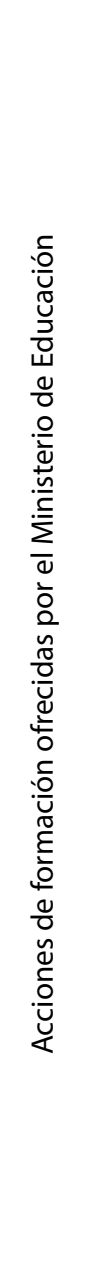 } & \multirow{4}{*}{ Acceso a la formación en servicio } & Acceso a la información - recepción de la información \\
\hline & & $\begin{array}{l}\text { Remite a las formas y mecanismos a través de los cuales los docentes tomaron } \\
\text { conocimiento o se enteraron de las ofertas de formación continua, como también } \\
\text { a la identificación de dificultades asociadas con las estrategias de comunicación } \\
\text { implementadas. Incluye asimismo la calidad, cantidad de la información y } \\
\text { oportunidad-momento en que el destinatario toma contacto. }\end{array}$ \\
\hline & & Condiciones de acceso y participación en las acciones formativas \\
\hline & & $\begin{array}{l}\text { Refiere a ciertos requisitos y atributos de las acciones formativas que operaron } \\
\text { desde la perspectiva de los participantes como facilitadores o no, para acceder } \\
\text { y participar. Cupo, asistencia a instancias presenciales, localización, fueron } \\
\text { algunos de los aspectos identificados. }\end{array}$ \\
\hline & \multirow{4}{*}{ Desarrollo de las acciones formativas } & Componentes pedagógico-didácticos de las acciones formativas \\
\hline & & $\begin{array}{l}\text { Remite a temática/s abordada/s, formatos (modalidad, actividades, materiales } \\
\text { de estudio, tiempos), desempeño de formadores, requisitos de evaluación/ } \\
\text { acreditación }\end{array}$ \\
\hline & & Condiciones para la permanencia-finalización en la acción formativa \\
\hline & & $\begin{array}{l}\text { Incluye aspectos que pueden operar como facilitadores u obstaculizadores para } \\
\text { el destinatario en el avance y finalización de la experiencia de formación iniciada, } \\
\text { tales como la localización, superposición con otros requerimientos, demandas } \\
\text { de la acción formativa (trabajos por presentar, lectura por realizar), obtención de } \\
\text { la certificación, entre otros aspectos. }\end{array}$ \\
\hline & $\begin{array}{l}\text { Apropiación - socialización de la } \\
\text { experiencia formativa }\end{array}$ & $\begin{array}{l}\text { Refiere a aquellas acciones diseñadas o ejecutadas por docentes participantes } \\
\text { de las instancias de formación continua, quienes reconocen haber puesto en } \\
\text { juego - de alguna manera- aportes de las propuestas de formación en su tarea } \\
\text { según la posición-cargo. Por ejemplo: en el aula, en el trabajo con colegas de la } \\
\text { institución, con otras instituciones-actores. }\end{array}$ \\
\hline \multirow{4}{*}{ 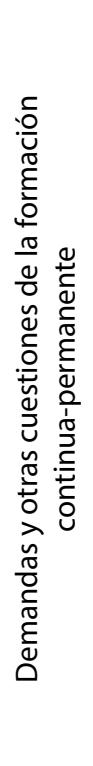 } & Demandas de formación en servicio & $\begin{array}{l}\text { Comprende aquellas áreas temáticas demandadas en futuras acciones } \\
\text { formativas que la población estudiada valora como relevantes a fin de fortalecer } \\
\text { capacidades para la mejora de sus prácticas docentes en el corto plazo, período } \\
2014-2015 \text {. }\end{array}$ \\
\hline & $\begin{array}{l}\text { Incorporación de nuevas tecnologías } \\
\text { de la información y la comunicación en } \\
\text { sus procesos de formación profesional }\end{array}$ & $\begin{array}{l}\text { Refiere a la identificación, consulta y valoración de la página web de la } \\
\text { Subsecretaría y de otros sitios virtuales en sus procesos de formación y desarrollo } \\
\text { profesional. }\end{array}$ \\
\hline & $\begin{array}{l}\text { Acceso - consulta de documentos y } \\
\text { materiales de lectura producidos o } \\
\text { sistematizados por el Ministerio }\end{array}$ & $\begin{array}{l}\text { Remite a la identificación de lectura de documentos, materiales, investigaciones } \\
\text { disponibles en la página web de la Subsecretaría de Estado de Promoción de } \\
\text { lgualdad y Calidad Educativa durante el período 2010-2012, como también su } \\
\text { importancia y aporte a sus práctica laborales, y de otras páginas oficiales de la } \\
\text { nación, de la provincia, de las direcciones de nivel, entre otros. }\end{array}$ \\
\hline & $\begin{array}{l}\text { Formación en servicio brindada por } \\
\text { otros oferentes }\end{array}$ & $\begin{array}{l}\text { Remite a experiencias formativas realizadas en el desarrollo profesional, ofertadas } \\
\text { por instituciones u organismos no dependientes del Ministerio de Educación, la } \\
\text { detección de posibles similitudes, diferencias y grado de significatividad para las } \\
\text { prácticas laborales de los destinatarios. }\end{array}$ \\
\hline
\end{tabular}

Fuente: La evaluación de acciones de formación docente continua desde la perspectiva de los destinatarios. Período $2010-2012$ (p. 8). 


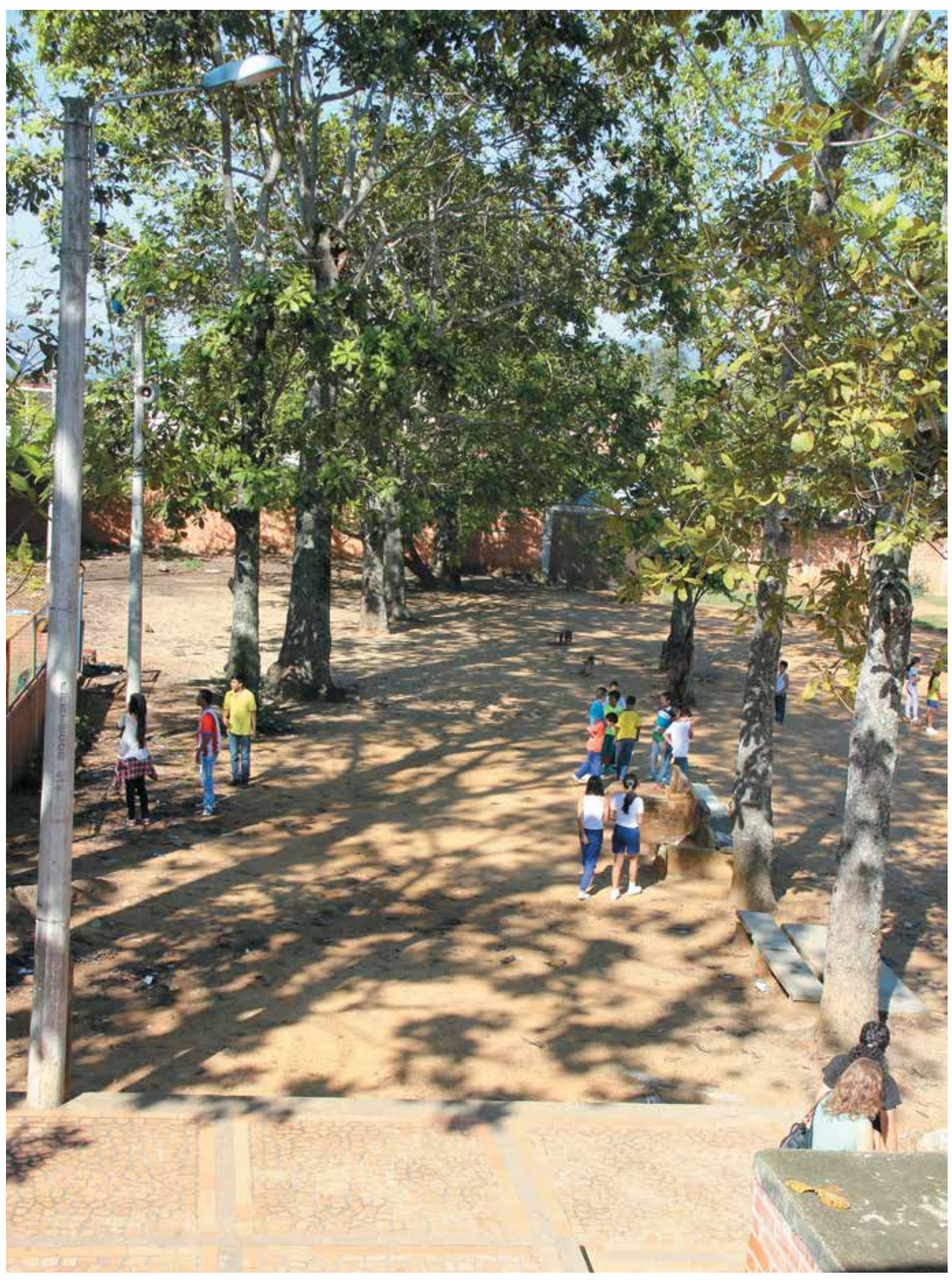


El tratamiento del material empírico fue realizado de acuerdo con el rol desempeñado por los participantes. Esto significa que hubo, en primer lugar, un tratamiento, sistematización y análisis de los datos a partir de la función de supervisor, director o docente ejercida por los participantes. Esta decisión metodológica se sostiene en el hecho de que el ejercicio diferente de funciones dentro del sistema educativo provincial otorga una perspectiva diferenciada a raíz de la especificidad del objeto de trabajo de cada rol. En otros términos, el cargo desempeñado por los participantes estructura sus puntos de vista y se ve materializado en sus percepciones y valoraciones de la oferta estudiada. El trabajo analítico de los datos buscó identificar recurrencias, diferencias y matices en los destinatarios acerca de las acciones formativas correspondientes al trienio 2010-2012.

El análisis de los datos también se orientó a identificar cambios percibidos por los destinatarios con respecto al período anterior. Dichos cambios fueron significados bajo tres posibles tipificaciones como una mejora, como un estancamiento o como un retroceso de las experiencias formativas en alguno de los planos indagados.

El trabajo analítico predijo también la generación de datos en torno a necesidades e intereses de desarrollo profesional, laboral para el corto plazo.

\section{Resultados}

Los resultados del estudio permitieron identificar ciertos rasgos y componentes como estructurantes en la generación de experiencias formativas con alta significación para los destinatarios. A continuación se presenta una síntesis de estos.

\section{Sobre el momento de acceso - incorporación a la acción formativa}

El primer momento de las experiencias formativas relacionado con el acceso a condicio- nes de inicio de la acción formativa, integra las valoraciones y percepciones de los destinatarios en torno a dos planos. Por un lado, la circulación y recepción de información por los canales y medios establecidos formalmente por las áreas centrales del Ministerio de la Provincia de Córdoba. Por otra, la generación de un circuito informal y autónomo entre pares que potencia el anterior. A partir de ello:

- Puede decirse que todos los participantes -más allá del cargo ocupado- valoraron positivamente los mecanismos utilizados para informar las propuestas a los potenciales destinatarios. Destacan como valiosa la instancia de difusión. Al respecto señalan como relevante la instancia de tomar contacto con información que permita caracterizar la propuesta formativa. Sin embargo, sugieren ampliar datos sobre la trayectoria académica, profesional y laboral de los responsables a cargo de su desarrollo, a fin de facilitar la toma de decisiones en torno a la elección de la oferta. Estos resultados presentan cierta continuidad con respecto a los obtenidos en el estudio 2008-2009.

- Dos piezas comunicacionales en soporte digital ocupan un lugar de relevancia en las valoraciones de los participantes. Se trata de la publicación de los procesos de circulación y recepción de información sobre las ofertas, en Novedades ${ }^{2}$ y el sitio web de la Secretaría de Estado de Promoción de Igualdad y Calidad Educativa ${ }^{3}$. El contacto directo del usuario del sitio -potencial destinatario de dicha oferta- con la descripción de las propuestas es vinculado a cierto proceso de "democratización" en el acceso a la información, especialmente para los docentes -quienes antes la recibían solo a través de la vía jerárquica y los

2 Es una publicación periódica en soporte digital que brinda información sobre la oferta que se llevará a cabo en un período determinado con ciertas descripciones sobre las características y alcances de cada acción formativa. Es realizada por la Subsecretaría de Estado de Igualdad y Calidad Educativa del Ministerio de Educación.

3 La dirección del sitio es www.igualdadycalidadcba.gov.ar 
canales formales establecidos para ello-. Sin embargo, este punto es relativizado por aquellos participantes que residen en el interior provincial y tienen dificultades para conectarse a Internet. En este sentido, es unánime la sugerencia de mantener la coexistencia de formas históricas de comunicación, a través de las direcciones de nivel y la vía jerárquica, junto a otras en soporte electrónico.

- El estudio ha permitido comprobar ciertas significaciones construidas en torno a la página web de la Subsecretaría de Igualdad y Calidad, que no solo se vincula con la recepción de la oferta disponible. Para muchos participantes es considerada como un espacio de consulta relevante para orientar, indagar, revisar y hasta reformular sus prácticas profesionales, así como para enriquecer sus repertorios de actuación. Al respecto, valoran variedad, pertinencia y actualidad de información, de publicaciones y de materiales publicados en el sitio.

- El aumento del número de acciones formativas ofrecidas junto a la diversificación de temáticas constituye para los consultados una fortaleza. En el marco de esa diversificación, es valorado el énfasis pedagógicodidáctico ofrecido por el Ministerio para acompañar a través de ciertas ofertas la implementación de los nuevos diseños curriculares en la Provincia, el tratamiento de contenidos transversales, la gestión y la evaluación, entre otros.

- Identifican como condiciones facilitadoras para acceder a instancias formativas, el aumento de acciones con modalidad distancia, acompañado de un mayor énfasis en la descentralización geográfica, con la incorporación de sedes distintas de las tradicionales, líneas sobre las cuales sugieren continuar. Se advierte un énfasis en la necesidad de precisar bien el sistema de transporte público como un componente clave en la localización de las sedes, por cuanto opera como determinante en las posibilidades reales de traslado de los potenciales destinatarios.

- Un cambio en la forma de inscripción para iniciar las acciones formativas marca con fuerza la lectura de los datos. En este sentido, el sistema de inscripción virtual implementado en el período estudiado 4 parece haber impactado significativamente en las prácticas de acceso a la oferta, que se ven diferenciadas según el cargo desempeñado por los participantes del estudio. En general, directores y docentes perciben la inclusión de esta modalidad como un avance al entender que amplía la autonomía en la toma de decisiones de los potenciales destinatarios para iniciar un proceso formativo. Un matiz distinto se perfila en el punto de vista de los participantes que se desempeñan como supervisores. Ellos observan dos situaciones. En primer término, perciben que la implementación de este sistema ha restringido las posibilidades de generar información sobre la dimensión de capacitación en las escuelas a cargo y, por lo tanto, en su propio ejercicio de funciones al ver restringida la tarea de acompañamiento por falta de información. En segundo término, si bien admiten las bondades de agilidad que ofrece el trámite virtualmente, observan que podría afectar al tomar las decisiones de acceder a una propuesta en función de las problemáticas o requerimientos institucionales regionales o zonales, no solo individuales. El estudio evidencia la complejidad en la toma de decisiones a la hora de iniciar o no una acción formativa. Desde este punto de vista pareciera constituirse en un particular momento que podríamos definir como crítico en tanto manifiesta tensiones entre intereses

4 Hasta ese momento las inscripciones eran realizadas por la vía jerárquica y se centralizaban -según el destinatario-en el director de la institución o supervisor de zona o región. 
y necesidades de distinto orden. Convergen aquellos de tipo personal y profesional de los destinatarios, los requerimientos y problemáticas de las instituciones donde se desempeñan y los que surgen del propio sistema educativo en su conjunto. Las sugerencias se inclinan por la coexistencia de formas de inscripción simultáneas. Distintas vías para resolver el trámite de matriculación favorecería el acceso a la oferta para contemplar las particularidades de las demandas y necesidades en la escala individual, institucional y regional-zonal; recursos de conectividad de las distintas zonasregiones de la provincia y cupos asignados.

- Los participantes del estudio visualizan, como otro punto de tensión, el requisito de asistir a instancias presenciales como parte del desarrollo de las acciones formativas y las consecuencias de su ausencia para resolver tareas propias de sus puestos de trabajo. Las necesidades de capacitarse y el desarrollo de sus obligaciones laborales cotidianas se debaten en un definido campo de tensiones.

\section{Sobre el momento de desarrollo de la experiencia formativa}

En relación con el desarrollo de la oferta, el estudio permitió identificar la convergencia de ciertos rasgos en aquellos dispositivos a los cuales los destinatarios otorgan significatividad en sus procesos de formación continua. Surge de los datos una suerte de tipología de aquellas propuestas conceptuadas como valiosas al contemplar -de manera explícita- al menos tres aspectos: la singularidad de la función que el participante desempeña dentro del sistema, la objetivación de prácticas y rutinas que permiten construir conocimiento situado $y$, finalmente, la revisión, diálogo y construcción de proyecto entre pares.

- Un rasgo que pareciera adquirir relevancia es aquel que incluye en las acciones for- mativas la revisión, reconstrucción de las prácticas laborales de los destinatarios, en función de la especificidad del cargo desempeñado. Convertir el hacer en objeto de análisis, en "contexto" o en "situación" es un camino fecundo para la construcción de conocimiento situacional de alta potencialidad para los destinatarios, aún con los límites que pudieran imponer los modos de formulación y circulación en que se producen (Ministerio de Educación de la Nación, 2011). Atender este atributo desde las políticas de formación permanente constituye un reto. Resolver la instancia de diseño de manera tal que atienda, a la vez, la escala del aula, de la institución, de la modalidad y del nivel, pareciera constituir un aspecto crítico. En este sentido, el estudio permitió advertir la complejidad de este rasgo, ya que se juegan tensiones entre dichos anclajes -individuales, profesionales e institucionales, locales- y la escala, más global, de los saberes ofrecidos en las instancias formativas desde las áreas centrales de diseño y planificación. Sobre este asunto Terigi advierte como posible riesgo el hecho de que

los procesos de formación ofrezcan a los docentes elementos para trabajar más sobre sus convicciones que sobre la organización de la enseñanza de acuerdo con esas convicciones y, más sobre las teorías que sobre los repertorios de prácticas y actuaciones concretas (2012, p. 52).

- Otro rasgo importante está vinculado con la presencia en las acciones formativas de cierto énfasis en la reflexión sistemática acerca de las rutinas, de manera tal que posibilite "objetivar" modos de hacer - saber, a partir de los cuales construir saber localmente situado y convertirlo en recursos potentes para la mejora en la identificación de una auténtica invención del hacer (Terigi, 2012, p.17). De acuerdo con lo expuesto, la oferta debería consolidar estos aspectos en el diseño de procesos 


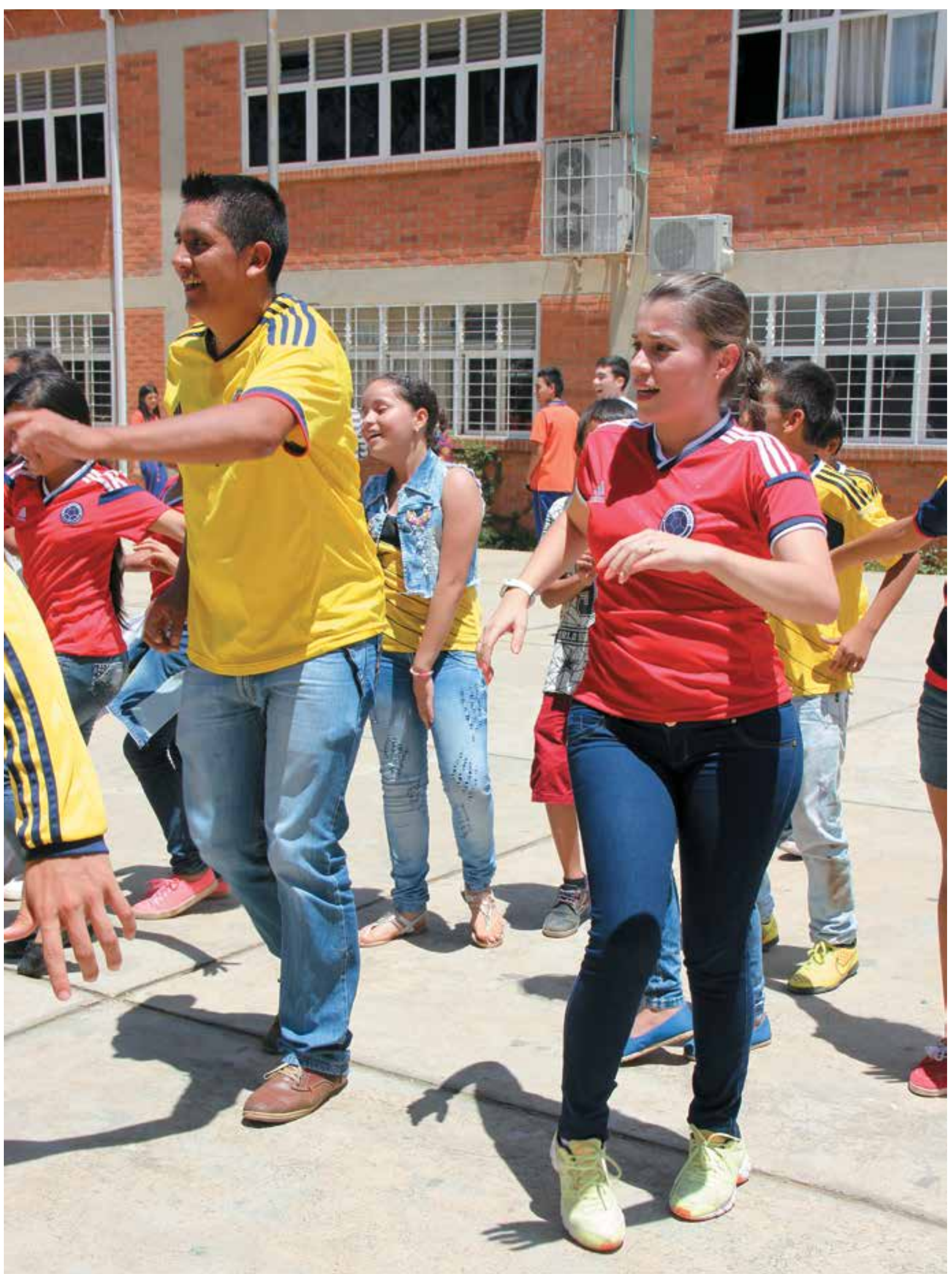


formativos permanentes que, a la vez, contemplen las trayectorias laborales y profesionales de los destinatarios. Cobra relevancia, junto a este rasgo, la inclusión en dichos procesos de instancias colaborativas entre pares. Esto es, promover desde las propuestas formativas el trabajo colegiado de la propia institución. Este hecho, pone en evidencia que "(...) los docentes enseñan en condiciones colectivas, y por ello la colaboración es una clave del desempeño profesional" (Terigi, 20102, p. 23). Lo dicho marca fuertemente, en los datos analizados, una conceptualización de la tarea profesional que traspasa el aula, como la construcción de un proyecto institucional entre pares, que demanda la participación de la mayoría de sus actores. Sobre este asunto Terigi, reconoce "(...) la complejidad del trabajo docente, su carácter político y su naturaleza institucional $y$, de suyo, colectiva" (2012, p. 11). Lo expresado implicaría la revisión de un componente que aparece en el análisis de la dimensión acceso ya como un punto problemático: cupo asignado por institución.

- El acompañamiento sostenido y sistemático con base en la orientación del destinatario junto a instancias de devolución personalizada de las producciones solicitadas constituyen prácticas clave para sostener la permanencia y finalización de procesos formativos.

- La revisión de las formas de entrega y distribución de las certificaciones que acreditan la aprobación del proceso formativo refiere centralmente como punto de convergencia al aludir en el momento de cierre de las acciones. Las sugerencias de los consultados ponen énfasis en agilizar esa instancia con el menor costo posible en tiempo y dinero, tanto para los destinatarios como para el Estado provincial.
Sobre el momento de apropiación - socialización de la experiencia

Los resultados permiten identificar tres aspectos que se hacen presentes e interactúan con los expuestos más arriba, para configurar condiciones favorables con respecto a la apropiación - socialización de experiencias formativas que a continuación se expone:

- Incorporar en el diseño de las acciones una instancia de seguimiento y orientación a cargo de equipos formadores junto a supervisores o directivos -según el caso- con posterioridad a la finalización de las acciones.

- Disponer de tiempos institucionalmente previstos para ello Esta valoración de los participantes no hace más que volver a poner en relieve la inscripción institucional del trabajo docente.

- La idea de sinergia es otra noción aludida por los participantes que consiste en las expectativas de que en las acciones formativas converjan, por un lado, necesidades y problemáticas institucionales del destinatario y, por otro, qué aborda y cuándo lo hace la oferta. Rasgo que adquiere mayor envergadura para los participantes en situaciones de cambio en el sistema, curriculares, por ejemplo.

Los rasgos expuestos, en términos globales, constituyen una línea de continuidad con resultados obtenidos en el estudio anterior.

\section{Acerca de demandas y necesidades de formación permanente para el próximo trienio}

Las demandas de formación identificadas para el corto plazo se centran especialmente en acompañar la situacionalidad de las prácticas laborales de los destinatarios en la implementación de cambios derivados de las políticas provinciales y federales. En este sentido la "(...) identificación de los distintos requerimientos 


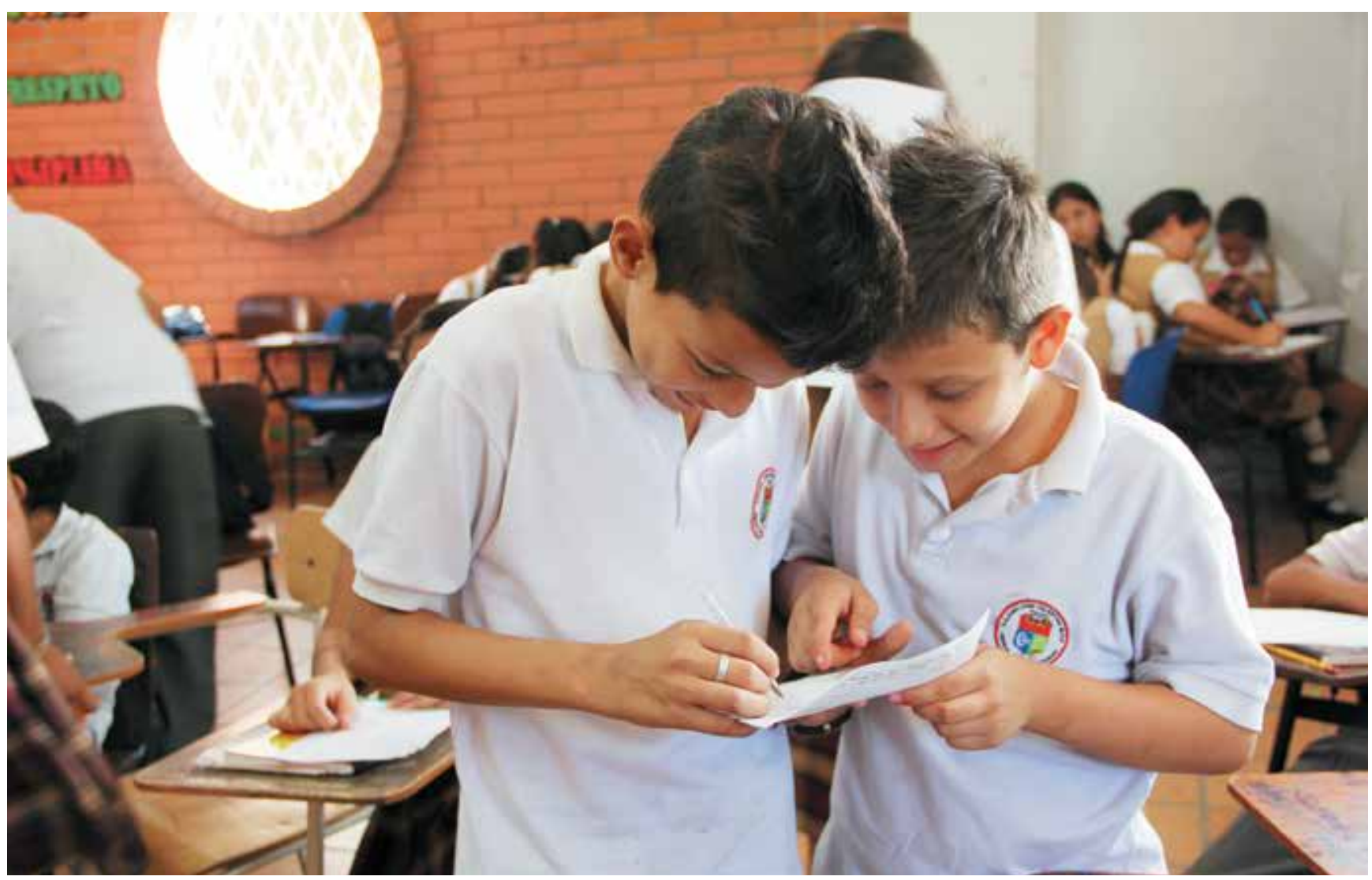

de formación que experimentan los docentes a lo largo de sus extensas carreras profesionales es una idea potente para seguir conceptualizando los procesos de formación en ejercicio" (Terigi, 2012, p. 53).

Un conjunto de temáticas fueron identificadas para el tratamiento en el próximo trienio, relevadas a partir de las problemáticas y necesidades expresadas por los consultados. En ese marco, sus expectativas están ligadas, a la vez, con "modos" de practicar procesos formativos. Se trata de afianzar -tal como se ha dicho a lo largo del trabajo- dispositivos de formación que pongan énfasis en acompañar la trayectoria laboral de los beneficiarios, atendiendo a los rasgos significativos detectados en las experiencias de los participantes del estudio.

\section{Conclusiones}

El trabajo realizado enfatiza en la necesidad de que las políticas de formación permanente orienten sus esfuerzos para diseñar accio- nes que contemplen de manera sustantiva los rasgos y componentes identificados con alto potencial para generar experiencias formativas significativas.

También requiere consolidar un camino iniciado que ajuste cada vez más la diversificación de las propuestas a partir de ciertas condiciones específicas de los destinatarios en función de los marcos institucionales y zonales - regionales de actuación.

En esta línea de exposición, interesa destacar la importancia de avanzar en un trabajo articulado en torno al diseño de la oferta futura en la Provincia que contemple, a la vez, dos planos: el de su producción, realizada en una escala global del sistema, y el de la recepción local por parte de los destinatarios. Establecer lazos más fluidos entre ambos planos es otro desafío que enfrentan hoy las políticas de formación docente continua (Ministerio de Educación de la Provincia de Córdoba, 2013). 
El estudio fue efectuado gracias al compromiso de las autoridades de cada nivel del Ministerio de Educación de la Provincia de Córdoba y de cada uno de los participantes quienes compartieron sus experiencias formativas.

Ante los resultados, surge el reto de consolidar aquellas acciones de formación que contribuyeron a fortalecer el trabajo y las prácticas escolares y, al mismo tiempo, mejorar aún más lo hecho. Es un reto que convoca a la labor aún más intensa en algunas líneas iniciadas. Pero obliga también a formular otras alternativas de desarrollo profesional.

Finalmente, interesa poner en valor el estudio al concebir la evaluación como una práctica que busca ir más allá de su concepción tradicional asociada al control. Pretende reponer dos planos centrales: el epistémico y el ético. Lo dicho supone concebirla como una práctica de pensamiento colectivo que oriente la toma de decisiones en el marco de la gestión de gobierno a partir de voces que se enlazan y le otorgan otro soporte. En este marco cobran envergadura, la formulación y desarrollo de propuestas de formación que atiendan a la vez necesidades en las escalas institucionales, locales y provinciales en articulación con las políticas educativas federales en Argentina.

\section{Referencias bibliográficas}

Fernández Ballesteros, R. (1996). Evaluación de programas. Una guía práctica en ámbitos sociales, educativos y de salud. Madrid: Síntesis.

Ministerio de Educación de la Nación Argentina (2011). Aportes pedagógicos a la reformulación de la formación inicial de los/as profesores/as de escuela secundaria en Argentina. Informe final. Buenos Aires, Argentina: Autor.

Ministerio de Educación de la Provincia de Córdoba. Subsecretaría de Promoción de Igualdad y Calidad Educativa (2010). Evaluación de acciones de formación docente continua desde la perspectiva de los destinatarios. Período 2008/2009. Córdoba, Argentina: Autor.
Ministerio de Educación de la Provincia de Córdoba. Subsecretaría de Promoción de Igualdad y Calidad Educativa (2013). Evaluación de acciones de formación docente continua desde la perspectiva de los destinatarios. Período 2010/2012. Córdoba, Argentina: Autor.

Mokate, K. (2000a). Convirtiendo el "monstruo" en aliado: La evaluación como herramienta de la gerencia social. Instituto Interamericano para el Desarrollo Social (INDES). Versión modificada. Recuperado el 28 de octubre de 2009, de http://www.bdp.org.ar/facultad/catedras/cp/analisispp/Mokate\%20evaluaci\%F3n.pdf

Mokate, K. (2000b). El monitoreo y la evaluación: herramientas indispensables de la gerencia social. Banco Interamericano de Desarrollo, Instituto Interamericano para el Desarrollo Social (INDES). Recuperado el 1 de octubre de 2009, de http://decon.edu. uy/100jovenes/materiales/sgNC-20.pdf

Nicastro, S. (2011). Revisitar la mirada sobre la escuela. Exploraciones acerca de lo ya sabido. Rosario: Homo Sapiens.

Nirenberg, O. (2010). Enfoques para la evaluación de políticas públicas. En Fioramonti, C. El estado y las políticas públicas en América Latina. Avances y desafíos de un continente que camina en el fortalecimiento de la inclusión social. Senado de la Provincia de Buenos Aires, Argentina. Recuperado de http://www.ceadel. org.ar/unicefiace/descargas/enfoques_eval_polpublicas_dic2010.pdf

Pichardo Muñiz, A. (1993). Evaluación del impacto social. Buenos Aires: Humanitas. Recuperado de http://miguelmartinezm.atspace.com/gruposfocales.html

Pinkasz, D., Montes, N., Marcalain, G., Legarralde, M., Dussel, l., Tiramonti, G. (2008). ¿Cómo se usa y qué impacto tiene la información empírica en el mejoramiento de los sistemas educativos en América Latina? Un estudio de caso en tres jurisdicciones de la Argentina. Informe Final de Investigación, Buenos Aires: Preal Flacso.

Terigi, F. (2012). Los saberes docentes. Formación, elaboración en la experiencia e investigación. VIII Foro Latinoamericano de Educación. Documento básico. Buenos Aires: Santillana. 